\title{
Noise-Induced Stop-and-Go Dynamics in Pedestrian Single-file Motion
}

\author{
Andreas Schadschneider ${ }^{1}$, Antoine Tordeux ${ }^{2}$ \\ ${ }^{1}$ Institut für Theoretische Physik, Universität zu Köln, 50937 Köln, Germany \\ as@thp.uni-koeln.de \\ ${ }^{2}$ Institut für Sicherheitstechnik, Bergische Universität Wuppertal, 42119 Wuppertal, Germany \\ tordeux@uni-wuppertal.de
}

\begin{abstract}
Stop-and-go waves are a common feature of vehicular traffic and have also been observed in pedestrian flows. Usually the occurrence of this self-organization phenomenon is related to an inertia mechanism. It requires fine-tuning of the parameters and is described by instability and phase transitions. Here, we present a novel explanation for stop-and-go waves in pedestrian dynamics based on stochastic effects. By introducing coloured noise in a stable microscopic inertia-free (i.e. first order) model, pedestrian stop-and-go behaviour can be described realistically without requirement of instability and phase transition. We compare simulation results to empirical pedestrian trajectories and discuss plausible values for the model's parameters.
\end{abstract}

Keywords: Pedestrian single-file motion, stop-and-go dynamics, first-order microscopic models, coloured noise, simulation

\section{Introduction}

Stop-and-go waves in traffic flow have been studied for several decades [1-3] (see [4,5] for reviews). Curiously, congested flows self-organise in waves of slow and fast traffic (stop-and-go) instead of streaming homogeneously. Stop-and-go dynamics which can not be explained by disturbance due to the infrastructure are observed in road traffic, bicycle and pedestrian movements [6] both in reality as well as in experiments [7]. Besides its scientific interest, such self-organisation phenomena impact transportation networks in terms of safety, economy, and comfort.

In modelling, stop-and-go behaviour can be analysed with microscopic, mesoscopic (kinetic) and macroscopic models based on non-linear differential systems (see for instance [8-10]), but also with discrete models like cellular automata. The models based on systems of differential equations have homogeneous equilibrium solutions that can become unstable for certain values of the parameters. The stable cases describe homogeneous dynamics whereas in unstable cases periodic or quasi-periodic solutions describe non-homogeneous dynamics. In the latter case, potentially stop-and-go waves can occur after fine-tuning of the parameters.

Phase transition and instability in self-driven dynamical systems far from equilibrium are commonly observed in physics, theoretical biology or social science [11-15]. In vehicular traffic, typical continuous models are inertial second order systems based on relaxation processes. Stop-and-go dynamics and matching to Korteweg-de Vries $(\mathrm{KdV})$ and modified $\mathrm{KdV}$ soliton equations occur when the inertia of the vehicles exceed critical values [8,16,17]. Empirical evidence for phase transitions in traffic, like hysteresis or capacity drop, has been observed in real data as well as during experiments [2,7]. Yet the number of phases and their characteristics remain actively debated [18].

Some studies describe pedestrian stop-and-go dynamics by means of, as traffic models, instability and phase transitions [19-22]. However, to our knowledge, empirical evidence for phase transitions and instability has not been observed for pedestrian flow. Pedestrian dynamics shows no pronounced inertia effect since human capacity nearly allows any speed variation at any time. Furthermore, pedestrian motion does not show mechanical delays. Nevertheless, stop-and-go behaviour is observed at congested density levels $[6,23]$. 
In this work, we propose a novel explanation of stop-and-go phenomena in pedestrian flows as a consequence of stochastic effects. We first present statistical evidence for the existence of Brownian noise in pedestrian speed time-series. Then a microscopic model composed of a minimal deterministic part for the convection and a relaxation process for the noise is proposed and analysed. Simulation results show that the stochastic approach allows describing realistic pedestrian stop-and-go dynamics without instability and fine-tuning of the parameters.

\section{Stochastic first order model}

Stochastic effects can have various roles in the dynamics of self-driven systems [24]. Generally speaking, the introduction of white noise in models tends to increase the disorder in the system [12] or prevents self-organisation [25]. Coloured noises can affect the dynamics and generate complex patterns $[26,27]$. Coloured noise has been observed in human response [28,29]. Pedestrian as well as driver behaviours result from complex human cognition. They are intrinsically stochastic in the sense that the deterministic modelling of the driving, i.e. the modelling of the human cognition composed of up to $10^{11}$ neurons [30], is not possible. Furthermore the behaviour of drivers and pedestrians can be influenced by various factors, e.g. environment, experience, culture, psychology, etc. Stochastic effects and noises are the main emphases of many pedestrian or road traffic modelling approaches (see, e.g., white noises [31, 17], pink-noise [32], action-point [33], or again inaccuracies or risk-taking behaviour [34,35]).

In Fig. 1 statistical evidence for the existence of Brownian noise in a time series of pedestrian speeds is presented. Brownian noise has a power spectral density (PSD) proportional to the square of the inverse noise frequency $1 / f^{2}$. A characteristic linear tendency is observed independent of the density (see [36] for further details). Such a noise with exponentially decreasing time-correlation function can be described by using the Ornstein-Uhlenbeck process (see e.g. [37]).
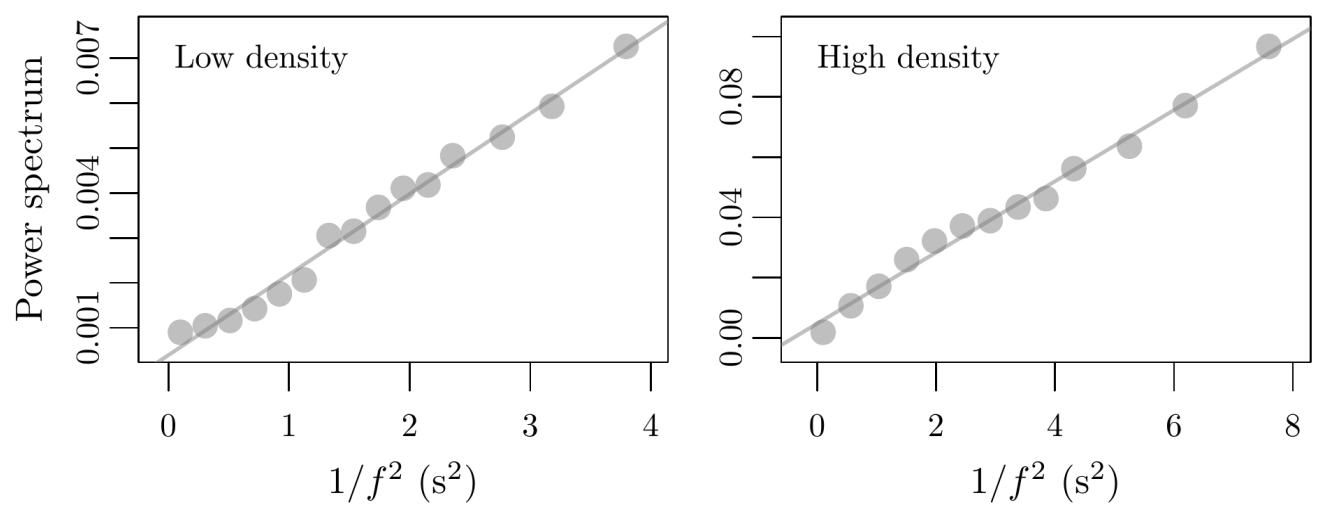

Fig. 1: Periodogram power spectrum estimate for the speed time-series of pedestrians at low and high density. The power spectrum shows the characteristic $1 / f^{2}$ behaviour of Brownian noise.

We denote in the following $x_{k}(t)$ the curvilinear position of the pedestrian $k$ at time $t$. Pedestrian $k+1$ is the predecessor of $k$. The model is the Langevin equation

$$
\begin{aligned}
& d x_{k}(t)=V\left(x_{k+1}(t)-x_{k}(t)\right) d t+\varepsilon_{k}(t) d t \\
& d \varepsilon_{k}(t)=-\frac{1}{\beta} \varepsilon_{k}(t) d t+\alpha d W_{k}(t)
\end{aligned}
$$

Here $V: s \rightarrow V(s)$ is a differentiable and non-decreasing optimal velocity (OV) function for the convection [8], while $\varepsilon_{k}(t)$ is a noise described by the Ornstein-Uhlenbeck stochastic process. In the following, an 
affine function $V(s)=(s-l) / T$ is used with $T$ the time gap between the agents and $l$ their size. $(\alpha, \beta)$ are positive parameters related to the noise. $\alpha$ is the volatility while $\beta$ is the noise relaxation time. $W_{k}(t)$ is a Wiener process. The homogeneous solution is stable for any positive values of the parameters in the deterministic case where $\alpha=0$. Note that alternatively, the model can be defined as a special stochastic form of the Full Velocity Difference model [38].

\section{Simulation results}

We have performed simulations using an explicit Euler-Maruyama numerical scheme with time step $\delta t=0.01 \mathrm{~s}$. The parameter values are $T=1 \mathrm{~s}, l=0.3 \mathrm{~m}, \alpha=0.1 \mathrm{~ms}^{-3 / 2}$ and $\beta=5 \mathrm{~s}$. Such values are close to the statistical estimates for pedestrian flow [36]. The length of the system is $L=25 \mathrm{~m}$, corresponding to the experimental situation, and the boundary conditions are periodic.

Simulations from jam initial conditions are carried out for systems with $n=25,50$ and 75 agents with the stochastic model and the unstable deterministic optimal velocity model introduced in [39]. The trajectories during the first $120 \mathrm{~s}$ are presented in Fig. 2 while the mean time-correlation functions for the distance spacing in stationary states (i.e. for large simulation time) are presented in Fig. 3. The peaks of the time-correlations match for both stochastic and deterministic models, i.e. the frequency of the stopand-go waves are the same. A wave propagates backward in the system at a speed $c=-l / T$ while vehicles travel in average at the speed $v=(L / n-l) / T$. In agreement with the theory, the wave period is $L /(v-c)=n T$.
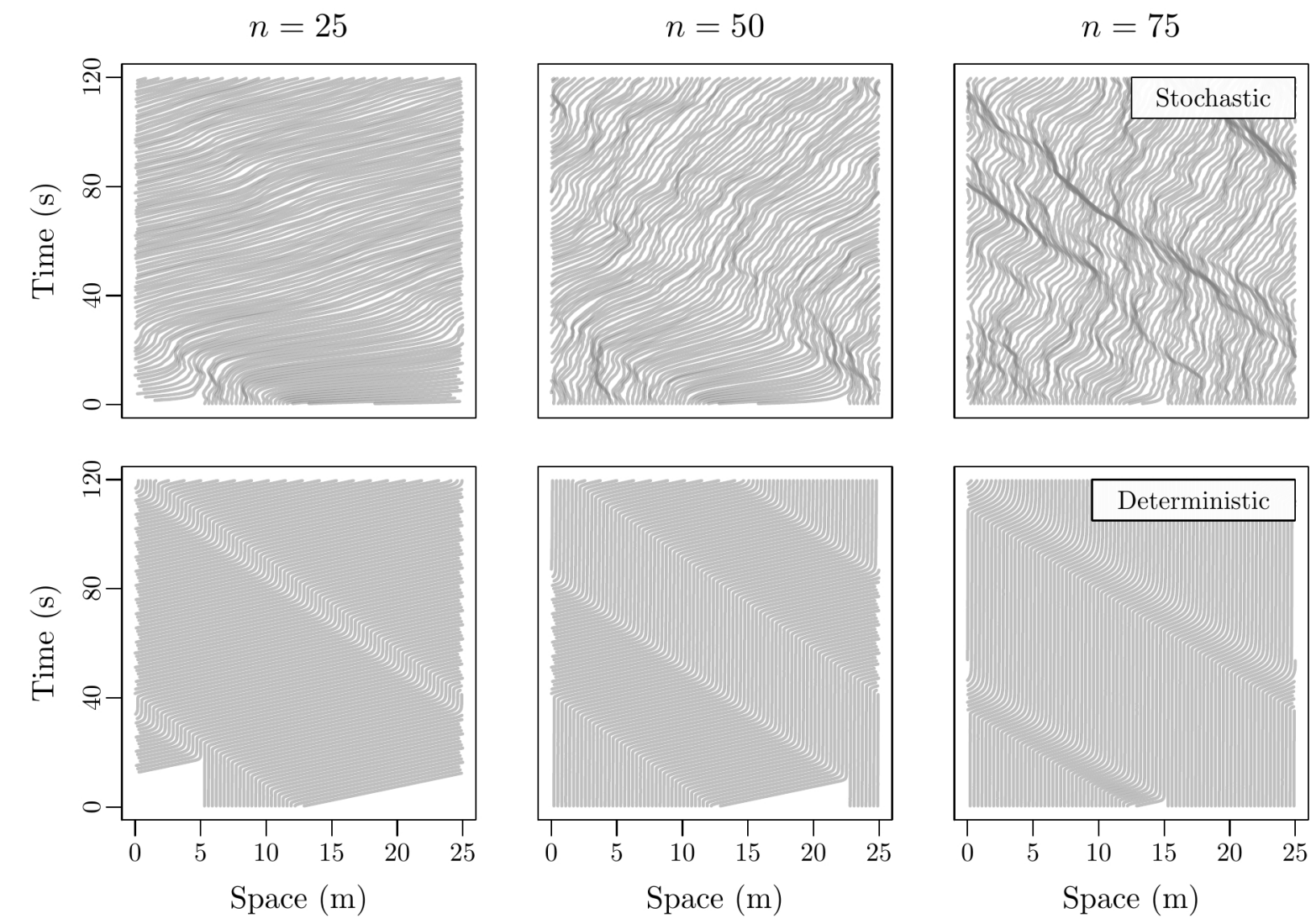

Fig. 2: Simulated trajectories for different density levels with the stochastic model presented in the paper (top panel) and the deterministic model with instability and phase transition introduced in [39] (bottom panels). 


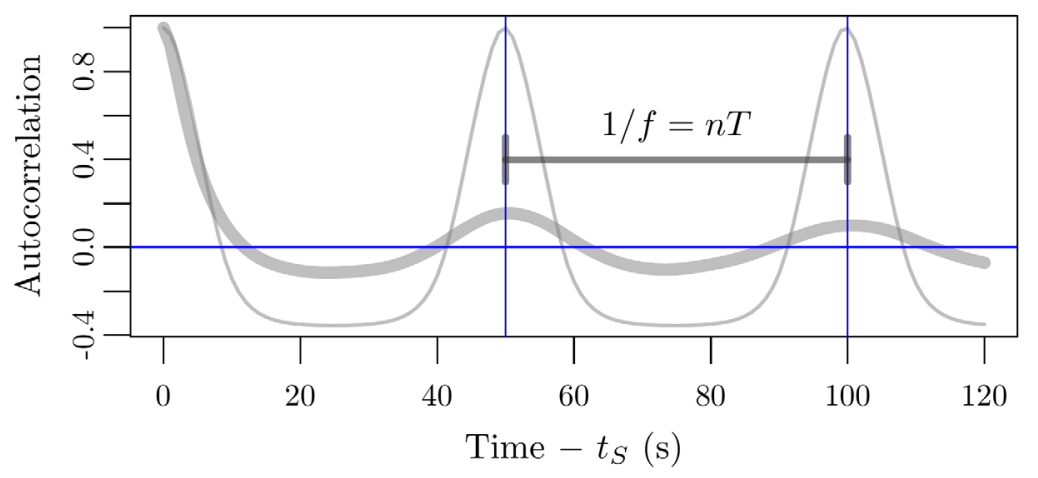

Fig. 3: Mean time-correlation function in stationary states of the distance spacing for the stochastic model (bold curve) and the deterministic model introduced in [39] (thin curve). The same period $1 / f=n T$ for the stop-and-go waves is observed. The simulation time to consider the system stationary is $t_{S}=2 * 10^{5}$.

Numerical experiments are carried out for different values of the noise parameters $\alpha$ and $\beta$. In Fig. 4 , the trajectories of 50 agents are presented for $\alpha=0.05,0.1$ and $0.2 \mathrm{~ms}^{-3 / 2}$ while $\beta=1.25,5$ and $20 \mathrm{~s}$. The values are chosen such that the amplitude of the noise is constant. For small $\beta$ the noise tends to be white and unstable waves emerge locally and disappear (see Fig. 4, left panel). For large relaxation times $\beta$, the noise autocorrelation is high and stable waves with large amplitude occur (Fig. 4, right panel). The parameters of the noise influence the amplitude of the time-correlation function, but not the frequency that only depends on the parameters $n$ and $T$, see Fig. 5.
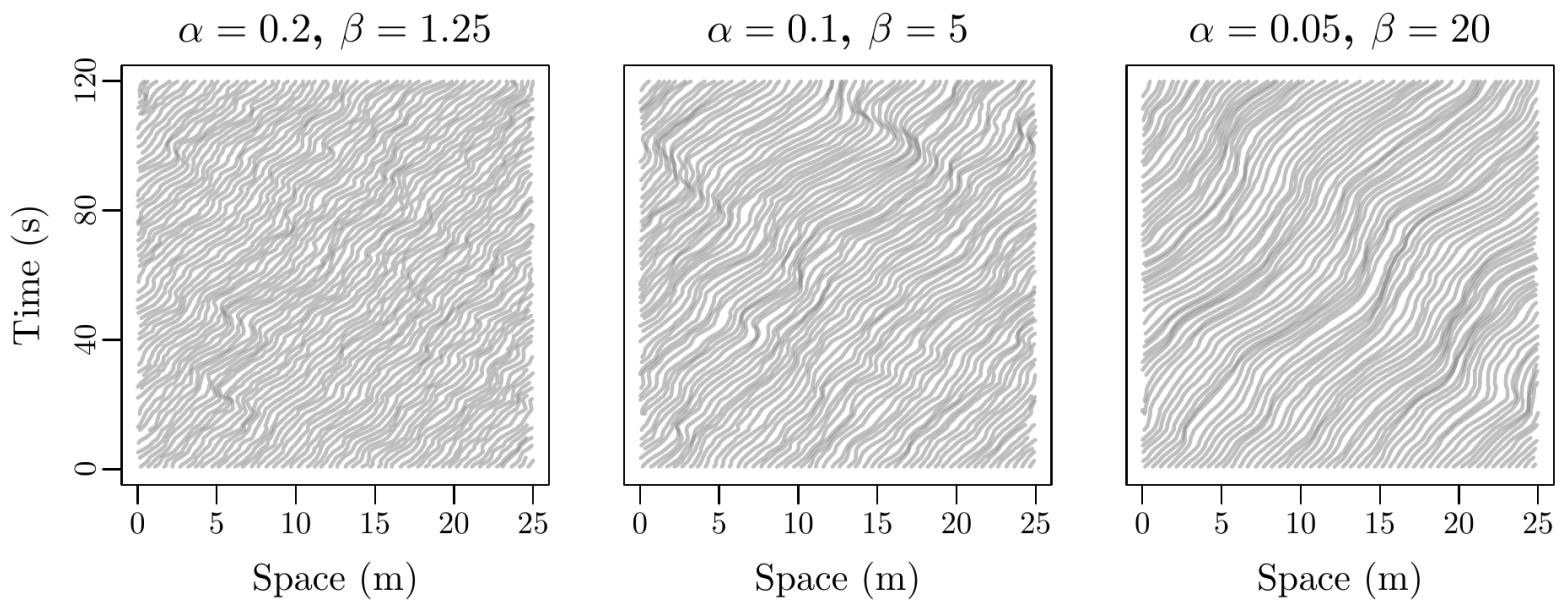

Fig. 4: Simulated trajectories for $n=50$ agents and different values of the noise parameters (units: $\alpha$ in $\mathrm{ms}^{-3 / 2}, \beta$ in s). The initial configuration is homogeneous. 


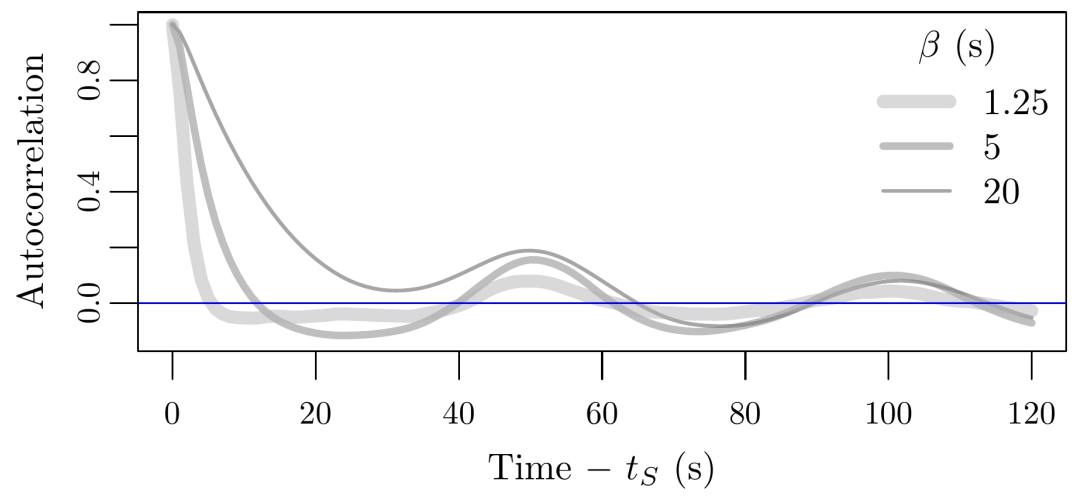

Fig. 5: Mean time-correlation function of the distance spacing in the stationary state for different values of the noise parameters. The frequency of the waves only depends on $n$ and $T$. The simulation delay time is $t_{S}=2 * 10^{5} \mathrm{~s}$.

In Fig. 6, real trajectories for experiments with 28, 45 and 62 participants (see [36]) and simulations with the stochastic model are compared. The simulations are in good agreement with the empirical data. Stop-and-go waves appear for semi-congested $(n=45)$ and congested $(n=62)$ states, while free states $(n=28)$ seem homogeneous in both empirical data and simulation.
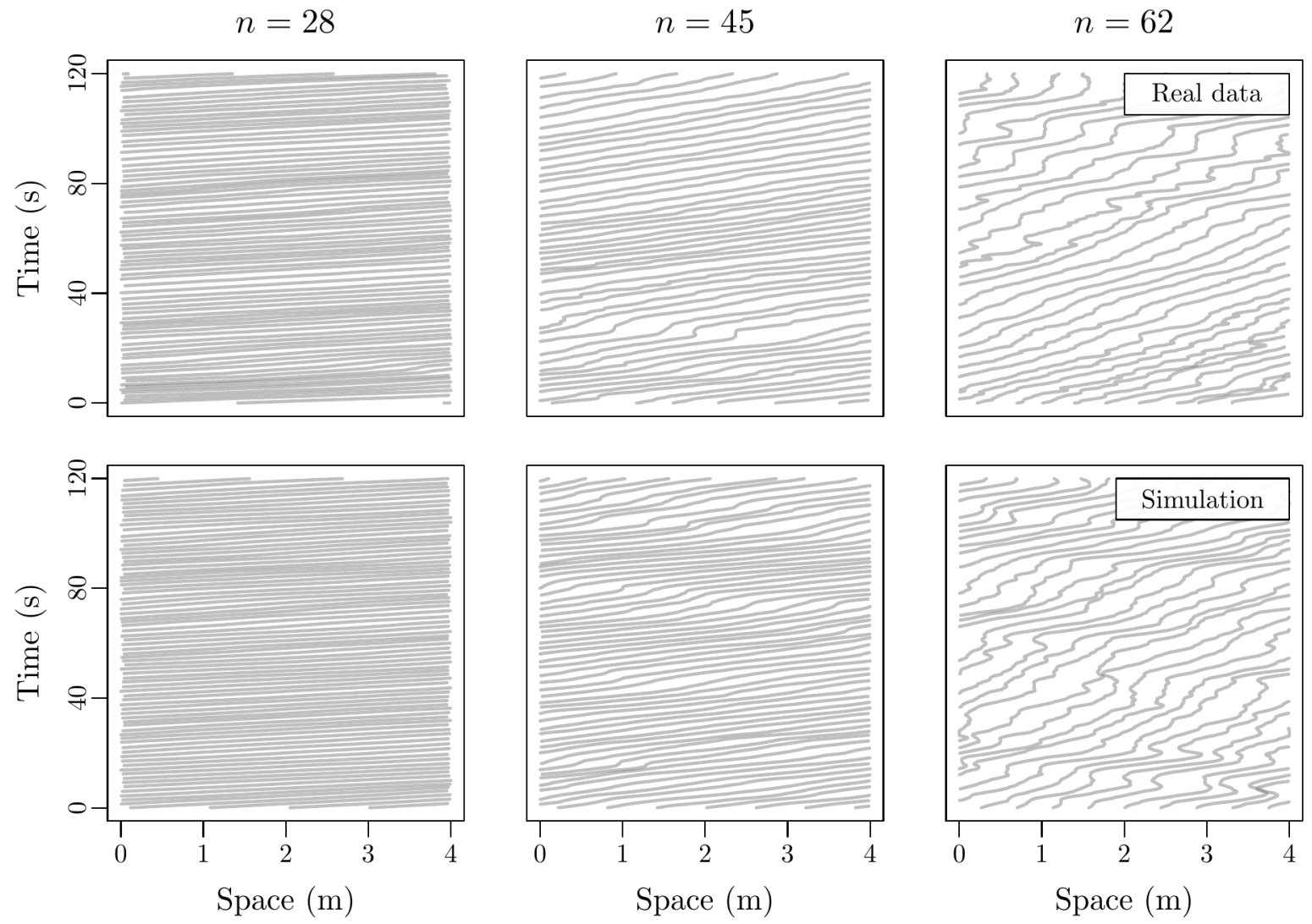

Fig. 6: Empirical (top panels) and simulated (bottom panels) trajectories for different densities. The initial configuration is homogeneous in both the real experiments and the simulations. 


\section{Conclusions}

We have proposed an alternative explanation for stop-and-go phenomena in pedestrian flows. In our stochastic approach, they are the consequence of a coloured noise in the dynamics of the speed. The oscillations in the system occur as a consequence of the perturbations introduced by the noise. This mechanism qualitatively describes stop-and-go waves, especially when the system is weakly damped. The approach differs from classical deterministic traffic models with inertia. Here stop-and-go occurs due to instability and phase transitions to periodical dynamics. These basic differences between the two routes to stop-and-go behavior are summarized in Fig. 7.
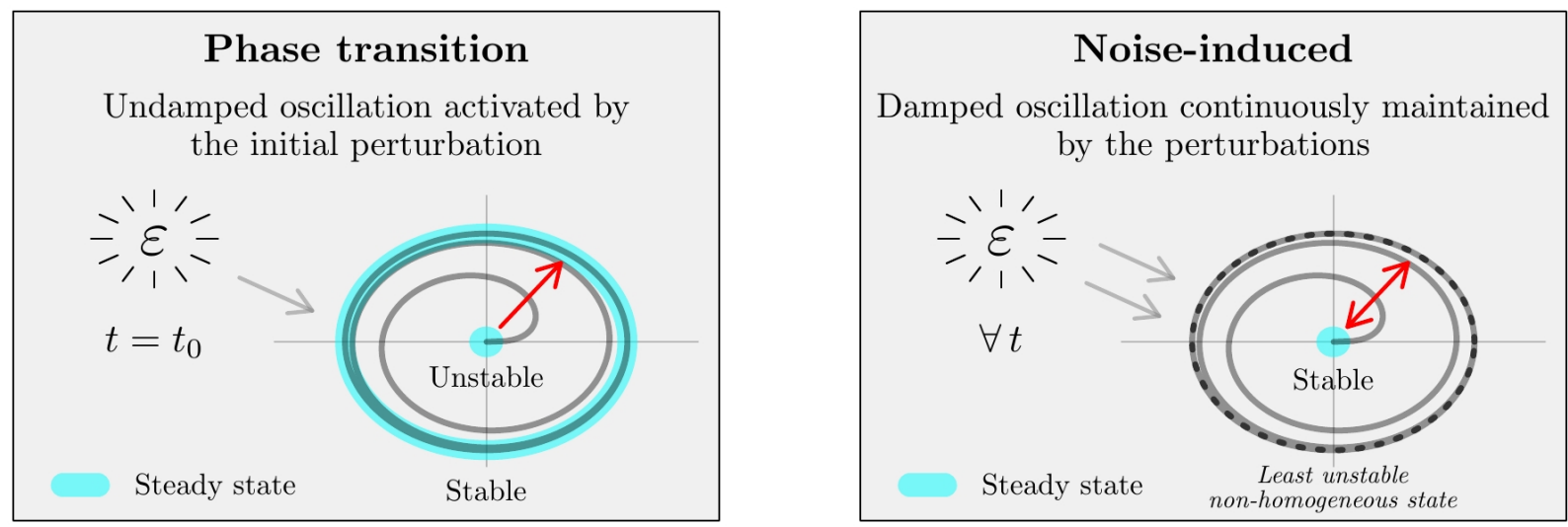

Fig. 7: Illustrative scheme for the modelling of stop-and-go dynamics with phase transition in the periodic solution (left panel) and the noise-induced oscillating behaviour (right panel).

We have identified two mechanisms based on relaxation processes for the description of stop-and-go waves. In the novel stochastic approach, the relaxation time is related to the noise and is estimated to approximately $5 \mathrm{~s}$ [36]. The parameter corresponds to the mean time period of the stochastic deviations from the phenomenological equilibrium state. Such a time can be large, especially when the deviations are small and the spacings are large. In the classical inertial approaches, the relaxation time is interpreted as the driver/pedestrian reaction time and is estimated by around 0.5 to $1 \mathrm{~s}$. Technically, such a parameter can not exceed the physical time gap between the agents (around 1 to $2 \mathrm{~s}$ ) without generating unrealistic (collision) behaviour and has to be set carefully.

The goal of the present study was to develop a realistic model for the description of pedestrian streams, but a toy model to demonstrate an alternative mechanism for the formation of stop-and-go waves. The results are relevant also for the interpretation of empirical and experimental data. In future studies, the effects of more realistic interactions, heterogeneities etc. need to be elucidated.

\section{Acknowledgements}

The authors thank Prof. Michel Roussignol for his help in the formulation of the model. Financial support by the German Science Foundation (DFG) under grant SCHA 636/9-1 is gratefully acknowledged. 


\section{References}

[1] Herman, R., Montroll, E., Potts, R., Rothery, R.: Traffic dynamics: analysis of stability in carfollowing. Op. Res. 7(1), 86-106 (1959).

[2] Kerner, B.S., Rehborn, H.: Experimental properties of phase transitions in traffic flow. Phys. Rev. Lett. 79, 4030-4033 (1997).

[3] Orosz, G., Wilson, R.E., Szalai, R., Stépán, G.: Exciting traffic jams: Nonlinear phenomena behind traffic jam formation on highways. Phys. Rev. E 80(4), 046,205 (2009).

[4] Chowdhury, D., Santen, L., Schadschneider, A.: Statistical physics of vehicular traffic and some related systems. Phys. Rep. 329(4-6), 199-329 (2000).

[5] Kerner, B.: The Physics of Traffic. Springer (2004).

[6] Zhang, J., Mehner, W., Holl, S., Boltes, M., Andresen, E., Schadschneider, A., Seyfried, A.: Universal flow-density relation of single-file bicycle, pedestrian and car motion. Phys. Lett. A 378(44), 3274 3277 (2014).

[7] Sugiyama, Y., Fukui, M., Kikushi, M., Hasebe, K., Nakayama, A., Nishinari, K., Tadaki, S.: Traffic jams without bottlenecks. Experimental evidence for the physical mechanism of the formation of a jam. New J. Phys. 10(3), 033,001 (2008).

[8] Bando, M., Hasebe, K., Nakayama, A., Shibata, A., Sugiyama, Y.: Dynamical model of traffic congestion and numerical simulation. Phys. Rev. E 51(2), 1035-1042 (1995).

[9] Helbing, D., Treiber, M.: Gas-kinetic-based traffic model explaining observed hysteretic phase transition. Phys. Rev. Lett. 81, 3042-3045 (1998).

[10] Colombo, R.: Hyperbolic phase transitions in traffic flow. SIAM J. Appl. Math. 63(2), 708-721 (2003).

[11] Ben-Jacob, E., Schochet, O., Tenenbaum, A., Cohen, I., Czirok, A., Vicsek, T.: Generic modelling of cooperative growth patterns in bacterial colonies. Nature 368, 46-49 (1994).

[12] Vicsek, T., Czirók, A., Ben-Jacob, E., Cohen, I., Shochet, O.: Novel type of phase transition in a system of self-driven particles. Phys. Rev. Lett. 75, 1226-1229 (1995).

[13] Bussemaker, H., Deutsch, A., Geigant, E.: Mean-field analysis of a dynamical phase transition in a cellular automaton model for collective motion. Phys. Rev. Lett. 78, 5018-5021 (1997).

[14] Buhl, J., Sumpter, D.J.T., Couzin, I.D., Hale, J.J., Despland, E., Miller, E.R., Simpson, S.J.: From disorder to order in marching locusts. Science 312, 1402-1406 (2006).

[15] Hermann, G., Touboul, J.: Heterogeneous connections induce oscillations in large-scale networks. Phys. Rev. Lett. 109, 018,702 (2012).

[16] Muramatsu, M., Nagatani, T.: Soliton and kink jams in traffic flow with open boundaries. Phys. Rev. E 60, 180-187 (1999).

[17] Tomer, E., Safonov, L., Havlin, S.: Presence of many stable nonhomogeneous states in an inertial car-following model. Phys. Rev. Lett. 84, 382-385 (2000).

[18] Treiber, M., Kesting, A., Helbing, D.: Three-phase traffic theory and two-phase models with a fundamental diagram in the light of empirical stylized facts. Transport. Res. B: Meth. 44(89), 9831000 (2010).

[19] Portz, A., Seyfried, A.: Modeling Stop-and-Go Waves in Pedestrian Dynamics. . In: Lect. Notes Comp. Sci., vol. 6068, pp. 561-568 (2010).

[20] Moussaïd, M., Helbing, D., Theraulaz, G.: How simple rules determine pedestrian behavior and crowd disasters. Proc. Nat. Acad. Sci. 108(17), 6884-6888 (2011).

[21] Kuang, H., Fan, Y., Li, X., Kong, L.: Asymmetric effect and stop-and-go waves on single-file pedestrian dynamics. Procedia Eng. 31, 1060 - 1065 (2012).

[22] Lemercier, S., Jelic, A., Kulpa, R., Hua, J., Fehrenbach, J., Degond, P., Appert-Rolland, C., Donikian, S., Pettr, J.: Realistic following behaviors for crowd simulation. Comput. Graph. Forum 31(2pt2), 489-498 (2012). 
[23] Seyfried, A., Portz, A., Schadschneider, A.: Phase coexistence in congested states of pedestrian dynamics. In: Lect. Notes Comp. Sci., vol. 6350, pp. 496-505 (2010).

[24] Hänggi, P., Jung, P.: Colored Noise in Dynamical Systems, pp. 239-326. John Wiley \& Sons, Inc. (2007).

[25] Helbing, D., Farkas, I., Vicsek, T.: Freezing by heating in a driven mesoscopic system. Phys. Rev. Lett. 84(6), 1240-1243 (2000).

[26] Arnold, L., Horsthemke, W., Lefever, R.: White and coloured external noise and transition phenomena in nonlinear systems. Z. Phys. B 29(4), 367-373 (1978).

[27] Castro, F., Sánchez, A.D., Wio, H.S.: Reentrance phenomena in noise induced transitions. Phys. Rev. Lett. 75, 1691-1694 (1995).

[28] Gilden, D., Thornton, T., Mallon, M.: $1 / f$ noise in human cognition. Science 267(5205), 1837-1839 (1995).

[29] Zgonnikov, A., Lubashevsky, I., Kanemoto, S., Miyazawa, T., Suzuki, T.: To react or not to react? Intrinsic stochasticity of human control in virtual stick balancing. J. R. Soc. Interface 11(99) (2014).

[30] Williams, R., Herrup, K.: The control of neuron number. Annu. Rev. Neurosci. 11(1), 423-453 (1988).

[31] Helbing, D., Molnár, P.: Social force model for pedestrian dynamics. Phys. Rev. E 51(5), 4282-4286 (1995).

[32] Takayasu, M., Takayasu, H.: 1/f noise in a traffic model. Fractals 01(04), 860-866 (1993).

[33] Wagner, P.: How human drivers control their vehicle. Eur. Phys. J. B 52(3), 427-431 (2006).

[34] Treiber, M., Kesting, A., Helbing, D.: Delays, inaccuracies and anticipation in microscopic traffic models. Phys. A 360(1), 71-88 (2006).

[35] Hamdar, S., Mahmassani, H., Treiber, M.: From behavioral psychology to acceleration modeling: Calibration, validation, and exploration of drivers cognitive and safety parameters in a risk-taking environment. Transp. Res. B-Meth. 78, 32 - 53 (2015).

[36] Tordeux, A., Schadschneider, A.: White and relaxed noises in optimal velocity models for pedestrian flow with stop-and-go waves. J. Phys. A 49(18), 185,101 (2016).

[37] Lindgren, G., Rootzen, H., Sandsten, M.: Stationary Stochastic Processes for Scientists and Engineers. Taylor \& Francis (2013).

[38] Jiang, R., Wu, Q., Zhu, Z.: Full velocity difference model for a car-following theory. Phys. Rev. E 64, 017,101 (2001).

[39] Tordeux, A., Seyfried, A.: Collision-free nonuniform dynamics within continuous optimal velocity models. Phys. Rev. E 90, 042,812 (2014). 